\title{
Exponential Operators For Generalized Lamb-Bateman Singular Integral Equations
}

\author{
Arman Aghili
}

(Communicated by Ravi P. AGARWAL)

\begin{abstract}
In this article, the author extends the application of exponential operators to solve certain non-linear fractional differential equation, space fractional partial differential equation and two dimensional LambBateman singular integral equation. Numerous constructive examples and exercises presented throughout the paper. The main purpose of this work is to present mathematical results that are useful to researchers in a variety of fields.
\end{abstract}

Keywords: Laplace transforms; Caputo fractional derivative; Parabolic Cylinder function; Riemann-Liouville fractional derivative; Lamb-Bateman singular integral equation.

AMS Subject Classification (2010): Primary: 26A33; Secondary: 44L05, 43A50

\section{Introduction}

In this study, the author present a general method of operational nature to obtain solutions for several types of singular integral equation, fractional differential equation, and partial differential equations with non - constant coefficients. Until now, two methods, have been more extensively used for solving PDEs, Laplace and Fourier transforms on the one hand and separation of variables on the other hand. Let us mention also solution in the form of a series of functions.

Definition 1.1. The Laplace transform of function $f(t)$ is defined as in [3]

$$
\mathcal{L}\{f(t)\}=\int_{0}^{\infty} e^{-s t} f(t) d t=: F(s) .
$$

If $\mathcal{L}\{f(t)\}=F(s)$, then $\mathcal{L}^{-1}\{F(s)\}$, is given by

$$
f(t)=\frac{1}{2 \pi i} \int_{c-i \infty}^{c+i \infty} e^{s t} F(s) d s,
$$

where $F(s)$ is analytic in the region $\operatorname{Re}(s)>c$.

Let us recall some important properties of the Laplace transform and useful Lemmas, that will be considered in the next part of this article.

Lemma 1.1. Let $\mathcal{L}\{f(t)\}=F(s)$ then, the following identities hold true.

Received : 30-05-2017, Accepted : 04-07-2017 
1. $\mathcal{L}^{-1}\left(e^{-k \sqrt{s}}\right)=\frac{k}{(2 \sqrt{\pi})} \int_{0}^{\infty} e^{-t \xi-\frac{k^{2}}{4 \xi}} d \xi$,

2. $e^{-\omega s^{\beta}}=\frac{1}{\pi} \int_{0}^{\infty} e^{-r^{\beta}(\omega \cos \beta \pi)} \sin \left(\omega r^{\beta} \sin \beta \pi\right)\left(\int_{0}^{\infty} e^{-s \tau-r \tau} d \tau\right) d r$,

3. $\mathcal{L}^{-1}\left(F\left(s^{\alpha}\right)\right)=\frac{1}{\pi} \int_{0}^{\infty} f(u) \int_{0}^{\infty} e^{-t r-u r^{\alpha} \cos \alpha \pi} \sin \left(u r^{\alpha} \sin \alpha \pi\right) d r d u$,

4. $\mathcal{L}^{-1}\left(F(\sqrt{s})=\frac{1}{2 t \sqrt{\pi t}} \int_{0}^{\infty} u e^{-\frac{u^{2}}{4 t}} f(u) d u\right.$.

Proof. See $[1,2]$.

Definition 1.2. The left Riemann-Liouville fractional derivative of order $0<\alpha<1$ is defined as following [2]

$$
D_{a}^{R L, \alpha} \phi(x)=\frac{1}{\Gamma(1-\alpha)} \frac{d}{d x} \int_{a}^{t} \frac{\Phi(\xi)}{(t-\xi)^{\alpha}} d \xi,
$$

it follows that $D_{a}^{R L, \alpha} \phi(x)$ exists for all $\Phi(t)$ belongs to $C[a, b]$ and $a<t<b$.

Note: A very useful fact about the R- L operators is that, they satisfy semi-group properties of fractional integrals. The special case of fractional derivative when $\alpha=0.5$ is called semi-derivative.

Definition 1.3. Let $0<\alpha<1$, the left Caputo fractional derivative of order $\alpha$ of $\phi(t)$ is defined as

$$
D_{a}^{c, \alpha} \phi(t)=\frac{1}{\Gamma(1-\alpha)} \int_{a}^{t} \frac{1}{(t-\xi)^{\alpha}} \phi^{\prime}(\xi) d \xi .
$$

Example 1.1. By using an appropriate integral representation for the modified Bessel function of the second kind of order $\nu, K_{\nu}(s)$, we show that

$$
\mathcal{L}^{-1}\left\{K_{0}(a \sqrt{s+\lambda}) K_{0}(b \sqrt{s+\psi})\right\}=\frac{e^{-\lambda t-\frac{a^{2}}{4 t}}}{2 t} \star \frac{e^{-\psi t-\frac{b^{2}}{4 t}}}{2 t},
$$

where $\star$ denotes the convolution operation, and

$$
\mathcal{L}^{-1}\left\{K_{0}^{2}(\beta \sqrt{s+\psi})\right\}=\int_{0}^{t} \frac{e^{\psi t-2 \psi \eta-\frac{\beta^{2} t}{4 \eta(t-\eta)}}}{4 \eta(t-\eta)} d \eta .
$$

Solution. It is well known (see [3]) that $K_{\nu}(a \sqrt{s})$ has the following integral representation

$$
K_{\nu}(a \sqrt{s})=\frac{(a \sqrt{s})^{\nu}}{2^{\nu+1}} \int_{0}^{\infty} e^{-\xi-\frac{a^{2} s}{4 \xi}} \frac{d \xi}{\xi^{\nu+1}} .
$$

At this point, using complex inversion formula for the Laplace transforms and the above integral representation we have

$$
\mathcal{L}^{-1}\left\{K_{0}(a \sqrt{s+\lambda})\right\}=\frac{1}{2 i \pi} \int_{c-i \infty}^{c+i \infty} \frac{e^{t s}}{2} \int_{0}^{\infty} e^{-\xi-\frac{a^{2}(s+\lambda)}{4 \xi}} \frac{d \xi}{\xi^{\eta+1}} d s .
$$

Changing the order of integration and simplifying to get

$$
\mathcal{L}^{-1}\left\{K_{0}(a \sqrt{s})\right\}=\int_{0}^{\infty} \frac{e^{-\xi-\frac{a^{2} \lambda}{4 \xi}}}{\xi}\left(\frac{1}{2 i \pi} \int_{c-i \infty}^{c+i \infty} \frac{e^{\left(t-\frac{a^{2}}{4 \xi}\right) s}}{2} d s\right) d \xi .
$$

The value of the inner integral is $\delta\left(t-\frac{a^{2}}{4 \xi}\right)$, we arrive at

$$
\mathcal{L}^{-1}\left\{K_{0}(a \sqrt{s+\lambda})\right\}=\int_{0}^{\infty} \frac{e^{-\xi-\frac{a^{2} \lambda}{4 \xi}}}{\xi} \delta\left(t-\frac{a^{2}}{4 \xi}\right) d \xi
$$


making the change of variable $t-\frac{a^{2}}{4 \xi}=u$, and using elementary properties of Dirac delta function, we get the following result

$$
\mathcal{L}^{-1}\left\{K_{0}(a \sqrt{s+\lambda})\right\}=\frac{e^{-\lambda t-\frac{a^{2}}{4 t}}}{2 t}
$$

Finally, we obtain

$$
\begin{aligned}
\mathcal{L}^{-1}\left\{K_{0}(a \sqrt{s+\lambda}) K_{0}(b \sqrt{s+\psi})\right\} & =\frac{e^{-\lambda t-\frac{a^{2}}{4 t}}}{2 t} \star \frac{e^{-\psi t-\frac{b^{2}}{4 t}}}{2 t} \\
& =\int_{0}^{t} \frac{e^{\lambda(t-\eta)-\frac{a^{2}}{4(t-\eta)}}}{2(t-\eta)} \frac{e^{-\psi \eta-\frac{b^{2}}{4 \eta}}}{2 \eta} d \eta,
\end{aligned}
$$

after simplifying, we arrive at

$$
\mathcal{L}^{-1}\left\{K_{0}(a \sqrt{s+\lambda}) K_{0}(b \sqrt{s+\psi})\right\}=\int_{0}^{t} \frac{e^{\lambda t-\eta(\lambda+\psi)-\frac{a^{2}}{4(t-\eta)}-\frac{b^{2}}{4 \eta}}}{4 \eta(t-\eta)} d \eta .
$$

Note: Let us consider the special case $a=b=\beta$ and $\lambda=\psi$, we get the following relation

$$
\mathcal{L}^{-1}\left\{K_{0}^{2}(\beta \sqrt{s+\psi})\right\}=\int_{0}^{t} \frac{e^{\psi t-2 \psi \eta-\frac{\beta^{2} t}{4 \eta(t-\eta)}}}{4 \eta(t-\eta)} d \eta .
$$

Lemma 1.2. Let us assume that $\mathcal{L}\{f(t)\}=F(s)$, then we have the following relations

1. $\mathcal{L}\left\{f\left(t^{2}\right)\right\}=\int_{0}^{\infty} \sqrt{\frac{1}{4 \pi \xi}} e^{-\frac{s^{2}}{4 \xi}} F(\xi) d \xi$,

2. $\mathcal{L}\left\{f\left(\frac{1}{t}\right)\right\}=\int_{0}^{\infty} \sqrt{\frac{\xi}{s}} J_{1}(2 \sqrt{s \xi}) F(\xi) d \xi$,

3. $\mathcal{L}\left\{f\left(t^{3}\right)\right\}=\int_{0}^{\infty} \sqrt{\frac{s}{\xi}} K_{\frac{1}{3}}\left(\left(\frac{s}{3 \sqrt[3]{\xi}}\right)^{\frac{2}{3}}\right) F(\xi) d \xi$.

Proof. See [3].

The above Lemma has immediate interesting applications as follows

Lemma 1.3. The following integral relations hold true.

1. $\int_{0}^{\infty} K_{\frac{1}{3}}\left(\left(\frac{1}{3 \sqrt[3]{\xi}}\right)^{\frac{2}{3}}\right)\left(\frac{\Gamma\left(\frac{1}{3}\right)}{3 \xi \sqrt{\xi} \sqrt[3]{\xi}}\right) d \xi=1$,

2. $\int_{0}^{\infty} \sqrt{\xi} J_{1}(2 \sqrt{\xi}) K_{0}(2 \sqrt{a \xi}) d \xi=\frac{1}{2(1+a)^{2}}$,

3. $\int_{0}^{\infty} \sqrt{\frac{1}{4 \pi \xi}} e^{-\frac{1}{4 \xi} \frac{\gamma+\ln \xi}{\xi}} d \xi=2 \gamma$.

Proof. (1) Let us take $f(t)=\sqrt[3]{t}$, then we get $F(s)=3 s^{-\frac{4}{3}} \Gamma\left(\frac{1}{3}\right)$, on the other hand we have

$$
\mathcal{L}\left\{f\left(t^{3}\right)\right\}=\mathcal{L}\{t\}=\frac{1}{s^{2}} .
$$

By setting all of the information of (3) from the Lemma 1.2, we infer

$$
\mathcal{L}\left\{f\left(t^{3}\right)\right\}=\frac{1}{3 \pi} \int_{0}^{\infty} \sqrt{\frac{s}{\xi}} K_{\frac{1}{3}}\left(\left(\frac{s}{3 \sqrt[3]{\xi}}\right)^{\frac{2}{3}}\right) \frac{\Gamma\left(\frac{1}{3}\right)}{3 \xi \sqrt[3]{\xi}} d \xi=\frac{1}{s^{2}} .
$$


Now, by choosing $s=1$ and after some simple manipulations we arrive at

$$
\int_{0}^{\infty} K_{\frac{1}{3}}\left(\left(\frac{1}{3 \sqrt[3]{\xi}}\right)^{\frac{2}{3}}\right) \frac{\Gamma\left(\frac{1}{3}\right)}{3 \xi \sqrt{\xi} \sqrt[3]{\xi}} d \xi=1 .
$$

(2) Let us take $f(t)=\frac{e^{-\frac{a}{t}}}{2 t}$, then we have $F(s)=K_{0}(2 \sqrt{a s})$. On the other hand $\mathcal{L} f\left(\frac{1}{t}\right)=\mathcal{L}\left(\frac{1}{2} t e^{-a t}\right)=\frac{1}{2(s+a)^{2}}$, using (2) from Lemma 1.2, we obtain

$$
\mathcal{L}\left\{f\left(\frac{1}{t}\right)\right\}=\int_{0}^{\infty} \sqrt{\frac{\xi}{s}} J_{1}(2 \sqrt{s \xi}) K_{0}(2 \sqrt{a \xi}) d \xi=\frac{1}{2(s+a)^{2}} .
$$

Now, by choosing $s=1$ and after simplifying, we arrive at

$$
\int_{0}^{\infty} \sqrt{\xi} J_{1}(2 \sqrt{\xi}) K_{0}(2 \sqrt{a \xi}) d \xi=\frac{1}{2(1+a)^{2}} .
$$

(3) Let us take $f(t)=\ln t$, then we have $F(s)=-\frac{\gamma+\ln s}{s}$, using (2) from the Lemma 1.2, we obtain

$$
\mathcal{L}\left\{f\left(t^{2}\right)\right\}=\int_{0}^{\infty} \sqrt{\frac{1}{4 \pi \xi}} e^{-\frac{s^{2}}{4 \xi}} \frac{\gamma+\ln \xi}{-\xi} d \xi=-2\left(\frac{\gamma+\ln s}{s}\right) .
$$

Now, by choosing $s=1$ and after simplifying, we arrive at

$$
\int_{0}^{\infty} \sqrt{\frac{1}{4 \pi \xi}} e^{-\frac{1}{4 \xi}} \frac{\gamma+\ln \xi}{\xi} d \xi=2 \gamma
$$

Lemma 1.4. Let us assume that $\mathcal{L}\{f(t)\}=F(s)$, then we have the following relation

$$
\mathcal{L}\left\{t^{\nu} f\left(t^{2}\right)\right\}=\frac{1}{4 \sqrt{\pi}} \int_{0}^{\infty} \xi^{\nu-2} e^{-\frac{s^{2} \xi^{2}}{2}} D_{\nu}(s \xi) F\left(\frac{1}{2 \xi^{2}}\right) d \xi .
$$

Proof. See [3].

The above Lemma has immediate interesting applications as below

Example 1.2. The following integral identity holds true

$$
\frac{1}{2 \sqrt{\pi}} \int_{0}^{\infty} \xi^{\nu-2} e^{-\frac{\xi^{2}}{2}-\frac{1}{2 \xi^{2}}} D_{\nu}(\xi) d \xi=e^{-1}
$$

Solution. Let us take $f(t)=\frac{1}{\sqrt{t}}$, then we have

$$
\mathcal{L}(f(t))=F(s)=\mathcal{L}\left(\frac{1}{\sqrt{t}}\right)=\sqrt{\frac{\pi}{s}} .
$$

At this point, we can evaluate $\mathcal{L}\left(t^{\nu} \sqrt{\frac{1}{t^{2}}}\right)$, in two different ways as follows. First, by definition of the Laplace transform we have

$$
\mathcal{L}\left(t^{\nu} \sqrt{\frac{1}{t^{2}}}\right)=\int_{0}^{+\infty} e^{-s t} t^{\nu-1} d t=\frac{\Gamma(\nu)}{s^{\nu}}
$$

Second, by using the Lemma 1.4 and denoting by $D_{\nu}$ the parabolic Cylinder function of order $\nu$, we get

$$
\mathcal{L}\left(t^{\nu} \delta\left(\frac{1}{t^{2}}-\lambda\right)\right)=\frac{1}{4 \sqrt{\pi}} \int_{0}^{\infty} \xi^{\nu-2} e^{-\frac{s^{2} \xi^{2}}{2}} D_{\nu}(s \xi) e^{-\frac{\lambda}{2 \xi^{2}}} d \xi
$$

Consequently, we obtain the following result

$$
\mathcal{L}\left(t^{\nu} \delta\left(\frac{1}{t^{2}}-\lambda\right)\right)=\frac{1}{4 \sqrt{\pi}} \int_{0}^{\infty} \xi^{\nu-2} e^{-\frac{s^{2} \xi^{2}}{2}} D_{\nu}(s \xi) e^{-\frac{\lambda}{2 \xi^{2}}} d \xi=\frac{1}{2} \lambda^{-\frac{\nu+3}{2}} e^{-\frac{s}{\sqrt{\lambda}}},
$$


after simplifying, we get the following relation

$$
\frac{1}{4 \sqrt{\pi}} \int_{0}^{\infty} \xi^{\nu-2} e^{-\frac{s^{2} \xi^{2}}{2}} D_{\nu}(s \xi) e^{-\frac{\lambda}{2 \xi^{2}}} d \xi=\frac{1}{2} \lambda^{-\frac{\nu+3}{2}} e^{-\frac{s}{\sqrt{\lambda}}} .
$$

In the above, let us choose $s=\lambda=1$, after simplifying, we obtain

$$
\frac{1}{2 \sqrt{\pi}} \int_{0}^{\infty} \xi^{\nu-2} e^{-\frac{\xi^{2}}{2}-\frac{1}{2 \xi^{2}}} D_{\nu}(\xi) d \xi=e^{-1} .
$$

Lemma 1.5. The following exponential identities hold true

1. $\exp \left( \pm \lambda \frac{d}{d t}\right) \Phi(t)=\Phi(t \pm \lambda)$,

2. $\exp \left( \pm \lambda t \frac{d}{d t}\right) \Phi(t)=\Phi\left(t e^{ \pm \lambda}\right)$,

3. $\exp \left(\lambda q(t) \frac{d}{d t}\right) \Phi(t)=\Phi(Q(F(t)+\lambda))$.

Where $F(t)$ is a primitive of $\frac{1}{q(t)}$, and $Q(t)=F^{-1}(t)$.

Proof. See $[5,6]$.

Lemma 1.6. The following exponential identity holds true

$$
\exp \left( \pm \frac{t^{2}}{\eta} \frac{d}{d t}\right) \Phi(t)=\Phi\left(\frac{\eta t}{t \mp \eta}\right)
$$

Proof. Let us take $t^{-1}=\xi$, then we have the following sequence of relations

$$
\begin{aligned}
\exp \left( \pm \frac{t^{2}}{\eta} \frac{d}{d t}\right) \Phi(t) & =\exp \left(\mp \frac{1}{\eta} \frac{d}{d \xi}\right) \Phi\left(\frac{1}{\xi}\right) \\
& =\phi\left(\frac{1}{\xi \mp \frac{1}{\eta}}\right) \\
& =\phi\left(\frac{1}{\frac{1}{t} \mp \frac{1}{\eta}}\right)=\phi\left(\frac{\eta t}{\eta \mp t}\right) .
\end{aligned}
$$

Lemma 1.7. The following exponential identity holds true for $1 \leq k \leq m-1$

$$
\left(\lambda-t^{2} \frac{d}{d t}\right)^{-\frac{k}{m}} \phi(t)=\frac{1}{\Gamma\left(\frac{k}{m}\right)} \int_{0}^{+\infty} \xi^{\frac{k}{m}-1} e^{-\lambda \xi} \phi\left(\frac{t}{1-t \xi}\right) d \xi .
$$

Proof. In order to show this identity, let us recall the following elementary integral relation

$$
s^{-\alpha}=\frac{1}{\Gamma(\alpha)} \int_{0}^{+\infty} \xi^{\alpha-1} e^{-s \xi} d \xi
$$

Let use the above integral with $s=\lambda-t^{2} \frac{d}{d t}, \alpha=\frac{k}{m}$, we get

$$
\left(\lambda-t^{2} \frac{d}{d t}\right)^{-\frac{k}{m}} \phi(t)=\frac{1}{\Gamma\left(\frac{k}{m}\right)} \int_{0}^{+\infty} \xi^{\frac{k}{m}-1}\left(e^{-\xi\left(\lambda-t^{2} \frac{d}{d t}\right)} \phi(t)\right) d \xi .
$$

Using the Lemma 1.6, we get

$$
\left(\lambda-t^{2} \frac{d}{d t}\right)^{-\frac{k}{m}} \phi(t)=\frac{1}{\Gamma\left(\frac{k}{m}\right)} \int_{0}^{+\infty} \xi^{\frac{k}{m}-1} e^{-\lambda \xi} \phi\left(\frac{t}{1-t \xi}\right) d \xi
$$


Example 1.3. Let us consider the following nonlinear fractional differential equation

$$
\sqrt[m]{\beta-t^{2} \frac{d}{d t}} y(t)=\phi(t)
$$

The above fractional differential equation has the following formal solution

$$
y(t)=\frac{1}{\Gamma\left(\frac{1}{m}\right)} \int_{0}^{+\infty} \xi^{\frac{1}{m}-1} e^{-\beta \xi} \phi\left(\frac{t}{1-t \xi}\right) d \xi .
$$

Solution. We can rewrite the above equation as follows

$$
y(t)=\left(\frac{1}{\sqrt[m]{\beta-t^{2} \frac{d}{d t}}}\right) \phi(t)=\left(\beta-t^{2} \frac{d}{d t}\right)^{-\frac{1}{m}} \phi(t),
$$

at this point, let us recall the following well - known identity

$$
a^{-\nu}=\frac{1}{\Gamma(\nu)} \int_{0}^{+\infty} e^{-a \xi} \xi^{\nu-1} d \xi, \quad \text { for } \nu>0
$$

Now, in the above integral we set $a=\beta-t^{2} \frac{d}{d t}$ and $\nu=\frac{1}{m}$ and infer

$$
\left(\beta-t^{2} \frac{d}{d t}\right)^{-\frac{1}{m}} \phi(t)=\frac{1}{\Gamma\left(\frac{1}{m}\right)} \int_{0}^{+\infty} \xi^{\frac{1}{m}-1}\left(e^{-\left(\beta-t^{2} \frac{d}{d t}\right) \xi} \phi(t)\right) d \xi
$$

Using again the Lemma 1.6 and after simplifying, we obtain the solution as follows

$$
y(t)=\frac{1}{\Gamma\left(\frac{1}{m}\right)} \int_{0}^{+\infty} \xi^{\frac{1}{m}-1} e^{-\beta \xi} \phi\left(\frac{t}{1-t \xi}\right) d \xi
$$

\section{Main Results}

In this section, we implement the exponential operator method to solve two partial differential equations with variable coefficients.

Problem 1. Let us consider the following initial value problem with the given initial condition

$$
\begin{aligned}
\frac{\partial u}{\partial t}+\lambda t^{\lambda-1} x^{2} \frac{\partial^{\alpha} u}{\partial x^{\alpha}} & =-\beta t^{\beta-1} u, \quad 0<\alpha, \beta, \lambda<1 . \\
u(x, 0) & =\phi(x) .
\end{aligned}
$$

for $t>0$ and $x \in \mathbb{R}$.

Solution. The above partial differential equation can be written as follows

$$
\frac{\partial u}{\partial t}=-\left(\lambda t^{\lambda-1}\left(x^{2} \frac{\partial^{\alpha}}{\partial x^{\alpha}}\right)+\beta t^{\beta-1}\right) u, \quad 0<\alpha, \beta, \lambda<1 .
$$

At this point, we solve the above first order differential equation with respect to variable $t$ and using initial condition to obtain

$$
u(x, t)=e^{-t^{\beta}} e^{-\left(\frac{x^{2}}{t-\lambda} \frac{\partial^{\alpha}}{\partial x^{\alpha}}\right)} \phi(x) .
$$

By using the second part of the Lemma 1.1, we find the result of the action of the exponential operator over the function. Therefore, we get the solution to PDE given as below

$$
\begin{aligned}
u(x, t) & =\frac{e^{-t^{\beta}}}{\pi} \int_{0}^{\infty} e^{-r^{\alpha}\left(t^{\lambda} x^{2} \cos \alpha \pi\right)} \sin \left(t^{\lambda} x^{2} r^{\alpha} \sin \alpha \pi\right)\left(\int_{0}^{\infty} e^{-r \tau} e^{-\tau \frac{\partial}{\partial x}} \phi(x) d \tau\right) d r \\
& =\frac{e^{-t^{\beta}}}{\pi} \int_{0}^{\infty} e^{-r^{\alpha}\left(t^{\lambda} x^{2} \cos \alpha \pi\right)} \sin \left(t^{\lambda} x^{2} r^{\alpha} \sin \alpha \pi\right)\left(\int_{0}^{\infty} e^{-r \tau} \phi(x-\tau) d \tau\right) d r .
\end{aligned}
$$


Note. Let us consider the special case $\alpha=0.5, \beta=2$ and $\phi(x)=\delta(x-c)$ with $c \in \mathbb{R}$, we get the following boundary value problem for $t>0$ and $x \in \mathbb{R}$

$$
\begin{aligned}
\frac{\partial u}{\partial t}+\lambda t^{\lambda-1} x^{2} \frac{\partial^{\frac{1}{2}} u}{\partial x^{\frac{1}{2}}} & =-2 t u, \quad \lambda>0 . \\
u(x, 0) & =\delta(x-c) .
\end{aligned}
$$

Using the relation (2.1), we get the following formal solution

$$
u(x, t)=\frac{e^{-t^{2}}}{\pi} \int_{0}^{\infty} \sin \left(t^{\lambda} x^{2} r^{0.5}\right)\left(\int_{0}^{\infty} e^{-r \tau} \delta(x-\tau-c) d \tau\right) d r .
$$

After simplifying, we obtain

$$
u(x, t)=\frac{x^{2} t^{\lambda} e^{-t^{2}-\frac{x^{4} t^{2 \lambda}}{4(x-c)}}}{2 \sqrt{\pi}(x-c)^{\frac{3}{2}}} .
$$

Problem 2. Let us consider the following generalized Lamb-Bateman singular integral equation in two dimensions where $\phi$ is the unknown function

$$
\int_{-\infty}^{+\infty} \int_{-\infty}^{+\infty} \frac{\phi\left(\xi-x^{2}-y^{2}\right)}{2 \pi \sqrt{x^{2}+y^{2}}} d x d y=g(\xi)
$$

The Lamb-Bateman singular integral equation was introduced to study the solitary wave diffraction and its solution was written in terms of an integral transform (see [4]).

Solution. Let us introduce the change of variable $x=r \cos \theta, y=r \sin \theta$, then (LBSIE) can be rewritten in the following form

$$
\int_{-\infty}^{+\infty} \int_{0}^{2 \pi} \frac{\phi\left(\xi-r^{2}\right)}{2 \pi \sqrt{(r \cos \theta)^{2}+(r \sin \theta)^{2}}} r d r d \theta=g(\xi) .
$$

or

$$
\int_{-\infty}^{+\infty} \phi\left(\xi-r^{2}\right) d r=g(\xi)
$$

Equivalently, we have

$$
\left(\int_{-\infty}^{+\infty} e^{-r^{2} \partial_{\xi}} d r\right) \phi(\xi)=g(\xi)
$$

The evaluation of the above integral leads to the following

$$
\sqrt{\frac{\pi}{\partial_{\xi}}} \phi(\xi)=g(\xi)
$$

in view of the Lemma 1.5, we arrive at the following relation

$$
\phi(\xi)=\sqrt{\frac{\partial_{\xi}}{\pi}} g(\xi) .
$$

At this point, in order to find the result of the action of fractional operator, we may re-write the above relation as follows

finally we get

$$
\phi(\xi)=\sqrt{\frac{1}{\pi}}\left(\partial_{\xi}\right)\left(\partial_{\xi}\right)^{-\frac{1}{2}} g(\xi) .
$$

The above is equivalent to

$$
\phi(\xi)=\frac{1}{\sqrt{\pi}} \partial_{\xi}\left(\frac{1}{\sqrt{\pi}} \int_{0}^{+\infty} d \eta \frac{1}{\sqrt{\eta}} e^{-\eta \partial_{\xi}} g(\xi)\right) .
$$

$$
\phi(\xi)=\frac{1}{\sqrt{\pi}} \int_{0}^{+\infty} \frac{1}{\sqrt{\eta}} g^{\prime}(\xi-\eta) d \eta
$$

Let us introduce the change of variable $\xi-\eta=w$, we get

$$
\phi(\xi)=\frac{1}{\sqrt{\pi}} \int_{-\infty}^{\xi} \frac{1}{\sqrt{\xi-w}} g^{\prime}(w) d w .
$$


Example 2.1. Let us solve the following two dimensional Lamb-Bateman singular integral equation with the given initial condition

$$
\int_{-\infty}^{+\infty} \int_{-\infty}^{+\infty} \frac{\phi\left(\xi-4 x^{2}-9 y^{2}\right)}{2 \pi \sqrt{4 x^{2}+9 y^{2}}} d x d y=J_{0}(\xi)
$$

Solution Using the change of variable $x=\frac{r}{2} \cos \theta, y=\frac{r}{3} \sin \theta$, the above equation rewrites as

or

$$
\int_{-\infty}^{+\infty} \int_{0}^{2 \pi} \frac{\phi\left(\xi-r^{2}\right)}{12 \pi \sqrt{(r \cos \theta)^{2}+(r \sin \theta)^{2}}} r d r d \theta=J_{0}(\xi) .
$$

$\int_{-\infty}^{+\infty} \phi\left(\xi-r^{2}\right) d r=6 J_{0}(\xi)$

Solving the above singular integral equation yields

$$
\phi(\xi)=\frac{6}{\sqrt{\pi}} \int_{-\infty}^{\xi} \frac{1}{\sqrt{\xi-w}} J_{0}^{\prime}(w) d w .
$$

Such that one gets finally

$$
\phi(\xi)=\frac{-6}{\sqrt{\pi}} \int_{-\infty}^{\xi} \frac{J_{1}(w)}{\sqrt{\xi-w}} d w .
$$

Remark. We can generalize the integral equation (LBSIE) to the form

$$
\int_{-\infty}^{+\infty} \int_{-\infty}^{+\infty} \ldots \int_{-\infty}^{+\infty} \frac{\phi\left(\xi-\mathbf{x}^{T} A \mathbf{x}\right)}{\sqrt{\left(\mathbf{x}^{T} \mathbf{x}\right)^{n-1}}} d x_{1} d x_{2} \ldots . . d x_{n}=g(\xi)
$$

where $\mathbf{x}=\left(x_{1}, x_{2}, \ldots \ldots, x_{n}\right)^{T}$ and $A$ is positive-definite.

\section{Conclusion}

Operational methods provide fast and universal mathematical tools for obtaining the solution of PDEs or even FPDEs. Combination of integral transforms, operational methods and special functions give more powerful analytical instruments for solving a wide range of engineering and physical problems. The paper is devoted to study exponential operators and their applications in solving certain boundary value problems. The main purpose of this work is to develop methods for solving two-dimensional Lamb-Bateman singular integral equation. We note that within such a new framework as we have described and developed in this article, the extensive usage of the integral transforms and exponential operator method opens up new and powerful possibilities, which be more deeply explored in the future publications.

\section{Acknowledgements}

The author would like to thank the referee/s for helpful comments.

\section{References}

[1] Aghili, A., New results involving Airy polynomials, fractional calculus and solution to generalized heat equation, New trends in mathematical sciences, 3 (2015).

[2] Aghili, A., Fractional Black-Scholes equation, International Journal of Financial Engineering, 4 (1) (2017) World Scientific Publishing Company, DOI: 10.1142 /S2 424786317500049.

[3] Apelblat, A., Laplace transforms and their applications, Nova Science Publishers Inc., New York, 2012.

[4] Babusci, D., Dattoli, G., Sacchetti, D., The Lamb--Bateman Integral Equation and the Fractional Derivatives, Fractional calculus and applied analysis, 14 (2) (2011), 317-320.

[5] Dattoli, G., Operational methods, fractional operators and special polynomials. Applied Mathematics and Computations, 141 (2003) 151-159.

[6] Dattoli, G., Srivastava, H.M., Zhukovsky, K.V., Operational methods and differential equations to initial value problems. Applied Mathematics and computations, 184 (2007) 979-1001. 


\section{Affiliations}

ARMAN AGILI

AdDRESS: Department of Applied Mathematics, Faculty of Mathematical Sciences, University of Guilan, P.O.Box, 1841, Rasht-Iran

E-MAIL: arman.aghili@gmail.com, armanaghili@yahoo.com 